\title{
Treatment of Non-Transplant Patients with Multiple Myeloma: Routine Treatment by Office-Based Haematologists in Germany - Data from the Prospective Tumour Registry Lymphatic Neoplasms (TLN)
}

\author{
Wolfgang Knauf ${ }^{a} \quad$ Wolfgang Abenhardt $^{b} \quad$ Ali Aldaoud ${ }^{c} \quad$ Arnd Nusch $^{d}$ Renate Grugel ${ }^{\mathrm{e}}$ \\ Michaela Münz ${ }^{e}$ Holger Hartmann ${ }^{e}$ Norbert Marschner ${ }^{f}$ on behalf of the TLN Study Group \\ ${ }^{a}$ Onkologische Gemeinschaftspraxis, Frankfurt/M., Germany \\ ${ }^{\mathrm{b}}$ Münchner Onkologische Praxis, München, Germany \\ ${ }^{c}$ Dr. Aldaoud - Dr. Schwarzer Forschungsgesellschaft mbH, Leipzig, Germany \\ ${ }^{\mathrm{d}}$ Praxis für Hämatologie und internistische Onkologie, Velbert, Germany \\ e iOMEDICO, Freiburg i.Br., Germany \\ ${ }^{f}$ Praxis für interdisziplinäre Onkologie und Hämatologie, Freiburg i.Br., Germany
}

\section{Keywords}

Multiple myeloma - Non-transplant patients .

Registries - Ambulatory care - Germany - Epidemiology . Drug therapy - Statistical and numerical data

\section{Summary}

Background: Various treatment options exist for patients with multiple myeloma (MM). Clinical registries provide insight into routine treatment and identify changes in treatment over time. Patients and Methods: The Tumour Registry Lymphatic Neoplasms (TLN) prospectively collects data on the treatment of patients with lymphoid B cell neoplasms as administered by office-based haematologists in Germany. Data on patient and tumour characteristics, comorbidities, systemic treatments and outcome parameters are recorded. Results: 371 non-transplant patients with MM were recruited between 2009 and 2011. At the start of first-line (second-line) treatment, the median age was 73 (75) years; $67 \%$ (74\%) of the patients had stage III MM (classification of Durie and Salmon) and $19 \%$ (28\%) had renal insufficiency. In the first line, $40 \%$ of the patients received bortezomib + melphalan + prednisone (VMP), $25 \%$ received bortezomib \pm dexamethasone $(V \pm D)$ and $8 \%$ were treated with melphalan + prednisone + thalidomide (MPT). While use of bortezomib-based regimens increased from $67 \%$ (2009) to $85 \%$ (2011), use of melphalan-based regimens decreased from $68 \%$ to $48 \%$. The overall objective response rate of treatment was $82 \%$. In the second line, $34 \%$ of the patients received $\mathrm{V} \pm \mathrm{D}$ and $16 \%$ lenalidomide + dexamethasone (LD). Conclusion: Bortezomib-based regimens dominate the first- and second-line treatment of MM. Future analyses will investigate outcome data, e.g. effectiveness of bortezomib retherapy compared to other second-line treatments.

\section{Introduction}

Multiple myeloma (MM) (International Classification of Diseases (ICD) C.90.0) is a haematological B cell malignancy characterised by the proliferation and expansion of abnormal monoclonal plasma cells and their infiltration of the bone marrow [1]. The disease is mostly accompanied by the secretion of non-functional clonal immunoglobulins, in some cases by clonal light chains only. Non-secretorial MM, however, is a rare variant. The disease may cause cytopenias with the need for substitute blood products and causes osteolytic lesions, osteoporosis, hypercalcaemia and renal insufficiency as the main symptomatic complications.

\section{KARGER}

Fax +497614520714

Information@Karger.com

www.karger.com (c) 2014 S. Karger GmbH, Freiburg Karger
$2296-5270 / 14 / 3711-0635 \$ 39.50 / 0$
Open access

This is an Open Access article licensed under the terms of the Creative Commons Attribution-NonCommercial 3.0 Unported license (CC BY-NC) (www.karger.com/OA-license), applicable to the online purposes only. 
MM is a rare cancer with an unfavourable prognosis. Amongst haematological malignancies, it represents the third most common cancer after non-Hodgkin's lymphoma (NHL) and leukaemia, respectively [2], and is thought to account for approximately 3,700 deaths in Germany in 2008 [3]. It is estimated that, in Germany, 6,000 patients per year are newly diagnosed with MM. The relative overall 5-year survival rate is only $40 \%$ [3]. It can be assumed that more than 30,000 patients experienced their first diagnosis with $\mathrm{MM}$ in the European Union in 2012 [4].

According to current guidelines, the CRAB criteria (i.e. hypercalcaemia, renal insufficiency, anaemia and bone disease) are used to decide on treatment initiation [5]. Patients without any CRAB criteria should be kept under active surveillance, whereas all others with symptomatic and advanced-stage disease should be treated systemically $[5,6]$.

For younger patients ( $<70$ years) and for the physically fit elderly, guidelines currently suggest induction therapy followed by high-dose chemotherapy with autologous peripheral blood stem cell transplantation (autoPBSCT) as first choice of treatment $[1,6,7]$. However, combination chemotherapy is the recommended treatment option in the presence of comorbidities that make high-dose chemotherapy intolerable. Local radiation may prevent fracture of osteolytic bone lesions and may control bone pain. Occasionally, solitary bone lesions may also be resected. Additional supportive therapy includes alleviation of pain, application of bisphosphonates (targeted against hypercalcaemia and bone disease and pain) and plasmapheresis (targeted against blood hyperviscosity).

In order to choose an adequate systemic treatment, multiple factors are to be considered, such as the stage of disease, the patient's biological age, comorbidities, and the patient's preferences. All these factors influence the decision-making process for inducing therapy.

With the advent of new chemotherapeutic treatment options - such as the proteasome inhibitor bortezomib and the immunomodulatory drugs lenalidomide and thalidomide treatment of MM has changed significantly over the last years.

In Germany, secondary ambulatory care is predominantly provided by office-based specialists [8]. Patients with haematological malignancies such as MM account for one of the largest proportions of patients treated by office-based haematologists in Germany. Haematological diseases account for a large proportion of diseases taken care of by office-based haematologists and oncologists [9]. Here, we present data from the Tumour Registry Lymphatic Neoplasms (TLN) established by and for German office-based haematologists. The focus of this paper is on the chemotherapeutic treatment of patients with MM who are not eligible for stem cell transplantation (SCT). It will illustrate the sociodemographic and clinical characteristics of patients with MM and their treatment in routine practice. The paper will show how the introduction of new substances has changed the treatment over time.

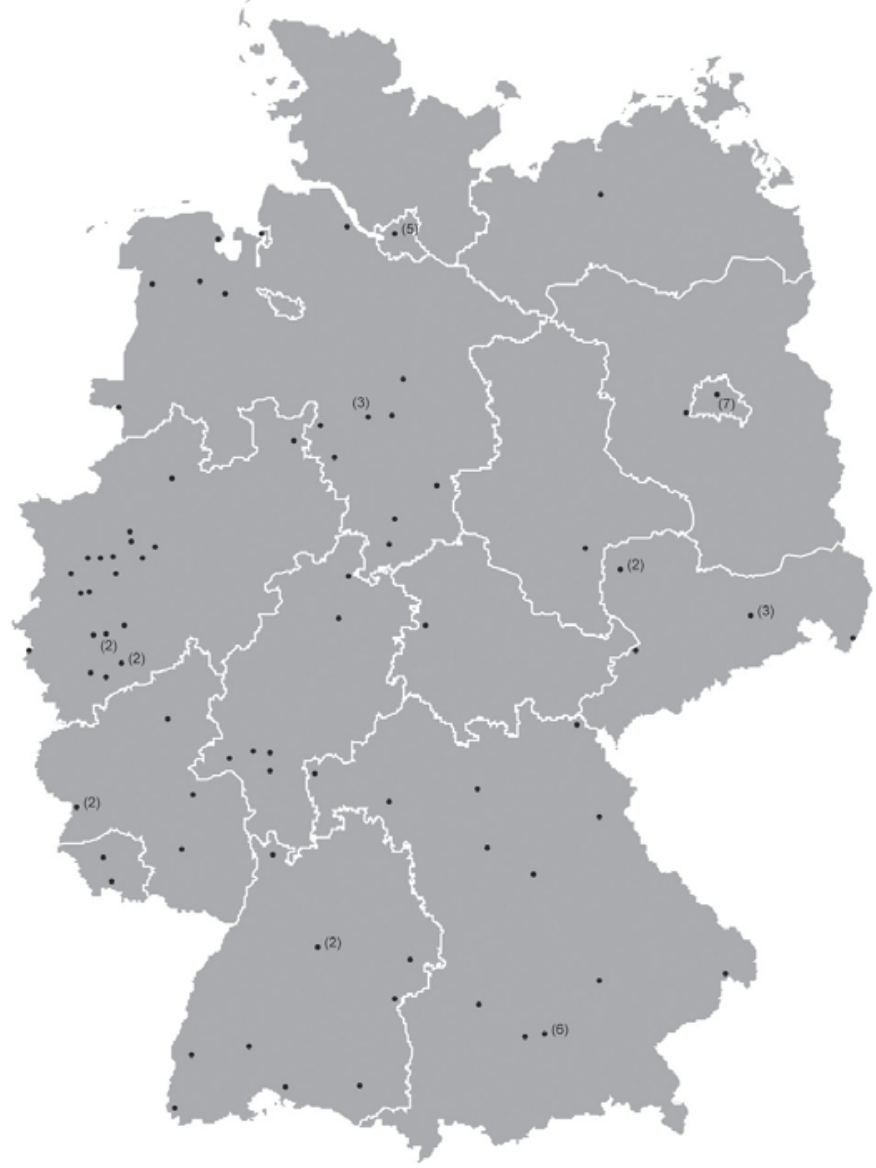

Fig. 1. Locations of the participating sites (Germany). Numbers in brackets represent the number of sites located in the same town.

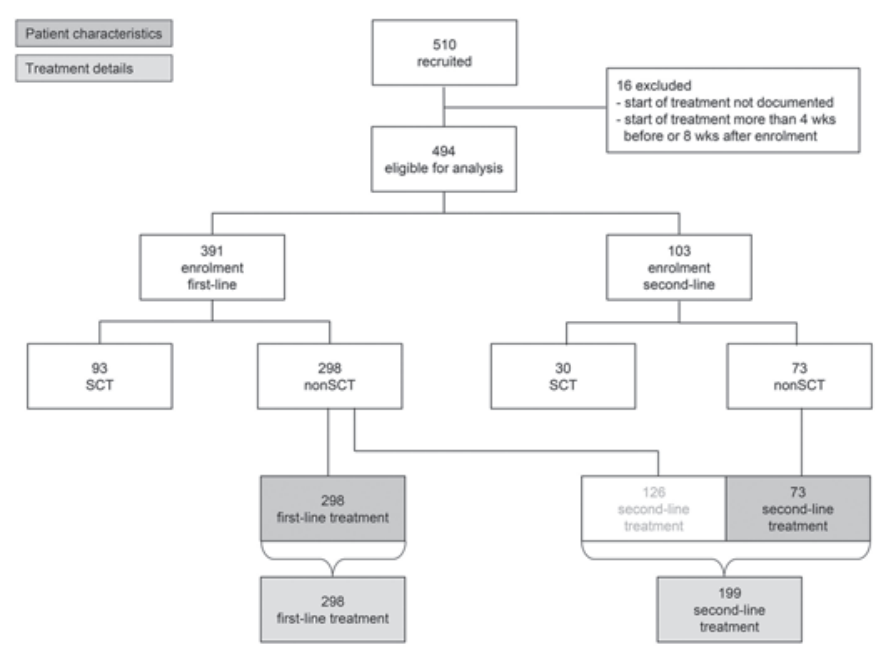

Fig. 2. Patient recruitment and treatment. Wks = Weeks.

\section{Patients and Methods}

The open, prospective, longitudinal, multicentre study 'Tumour Registry Lymphatic Neoplasms (TLN)' conducted by iOMEDICO in collaboration with the Arbeitskreis Klinische Studien (AKS) and the 


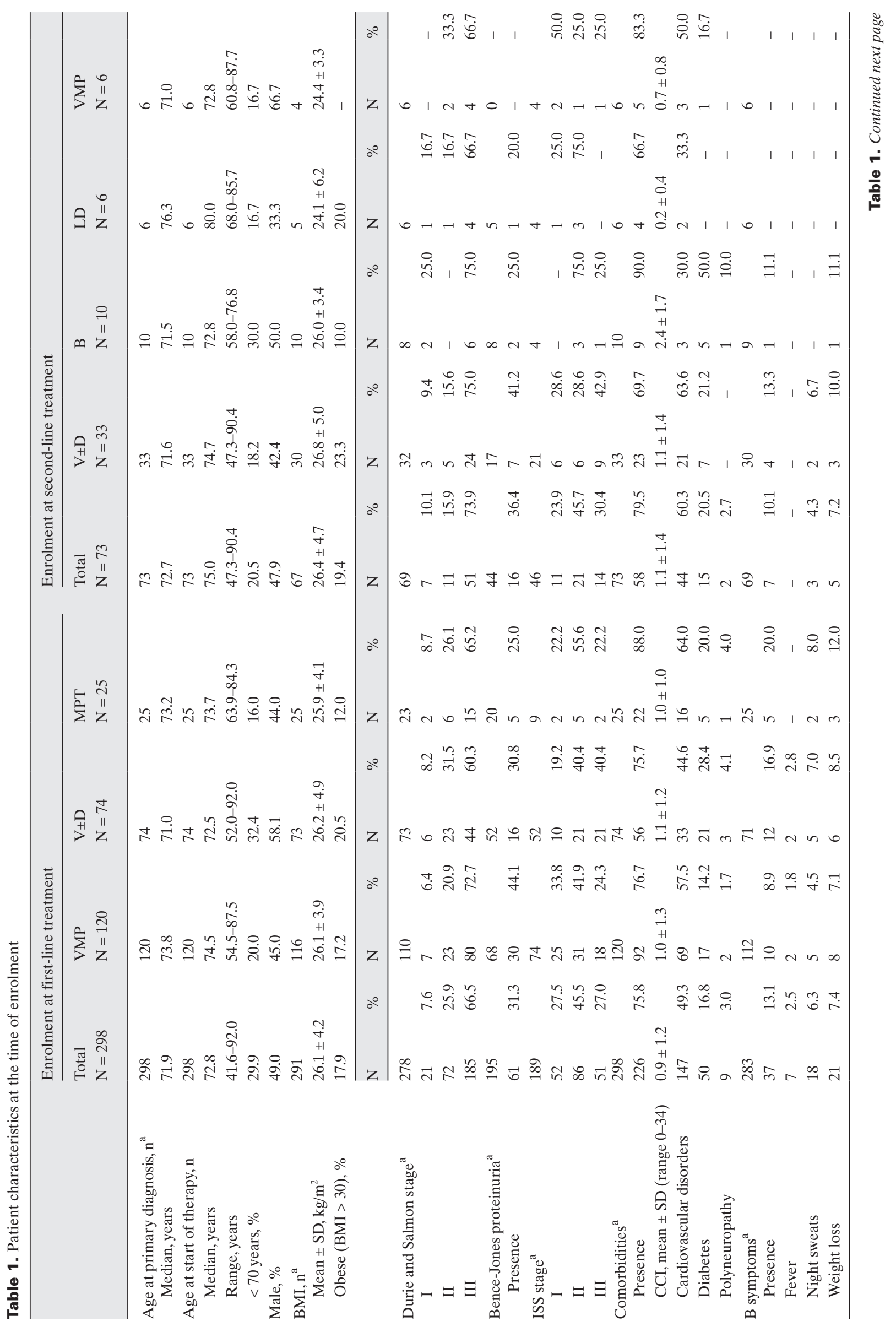




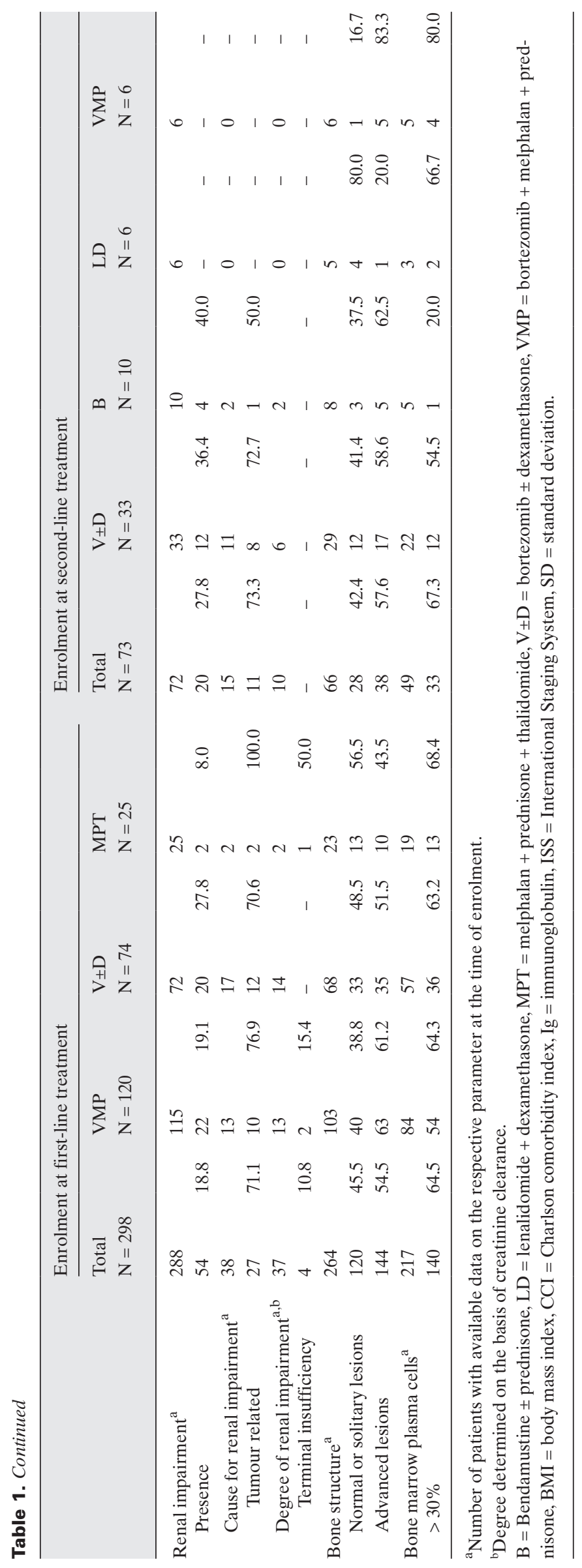

Kompetenznetz Maligne Lymphome (KML) was established in 2009 to provide insight into the current treatment of lymphatic neoplasms by German office-based haematologists. The study was reviewed by an ethics committee and is registered with the ClinicalTrial.gov registry (NCT00889798)

This clinical registry aims to recruit a total of 3,750 patients with aggressive or indolent NHL, chronic lymphocytic leukaemia (CLL) or MM. By the end of 2008, all members of the Association of Haematologists and Oncologists in Private Practice in Germany (BNHO e.V., in total 515 at that time) were asked to participate in the registry.

The TLN started recruiting in May 2009. By August 2013 (date of present analysis), a total of 3,383 patients were recruited by 115 sites. Recruitment of the MM subgroup finished in November 2011 when the targeted limit of 500 patients was attained. The distribution of the study sites across Germany is shown in figure 1. Overall, 262 physicians were recruiting patients, accounting for approximately over $30 \%$ of all officebased specialists within the field of haematology and oncology in Germany [8].

Patients of $\geq 18$ years at the start of their first- or second-line treatment were eligible for enrolment. Study centres had to enrol patients consecutively to ensure unselected recruitment of patients. Treatment of patients started within 4 weeks prior or until 8 weeks after signing the informed consent.

At the time of enrolment, data on the patients' sociodemography, tumour history, other clinical parameters as well as concomitant disorders are documented. Comorbidity is assessed using the Charlson comorbidity index (CCI) [10]. During the course of therapy, all systemic antineoplastic treatments (medication type and duration) as well as radiotherapies and/ or surgeries are documented. Treatment outcome parameters include best (clinical) tumour response(s), time(s) to progression(s) and time of death by any cause. Data are transferred from the patients' medical records to a secure web-based electronic case report form (eCRF) by the physicians or trained study nurses. Data are updated at any examination or change in therapy, or at least every 6 months. Automated plausibility controls and queries by the eCRF software, monitoring and regular completeness and plausibility checks by the data management ensure the reliability of the database.

Patients are treated according to the physicians' choice based on the patients' individual needs and schedules. No specifications are imposed on the physicians' assessment for treatment at any time. All patients are followed and documented for 5 years from enrolment (or until death, loss to follow-up or withdrawal of consent).

Progression-free survival (PFS) and overall survival (OS) were estimated with the Kaplan-Meier method [11]. PFS was defined as the interval between start of first-line therapy and the date of progression or death from any cause before the start of second-line therapy. Patients without such event were censored at either the start of second-line treatment, last contact or last documentation. OS was defined as the interval between start of first-line therapy and the date of death from any cause. Patients still alive were censored at the last documented date when they were known to be alive.

The median follow-up time was calculated according to the KaplanMeier estimate of potential follow-up, also termed 'reversed KaplanMeier' [12].

This analysis is based on 371 non-transplant patients with MM recruited by 83 sites between May 2009 and November 2011. Data on sociodemographic and clinical characteristics, documented at the beginning of the respective treatment, are available for 298 first-line and 73 second-line patients. Data on treatment are available for 298 first-line and $199 \mathrm{sec}-$ ond-line treatments because 126 of the first-line patients have already received a second-line treatment (fig. 2). 


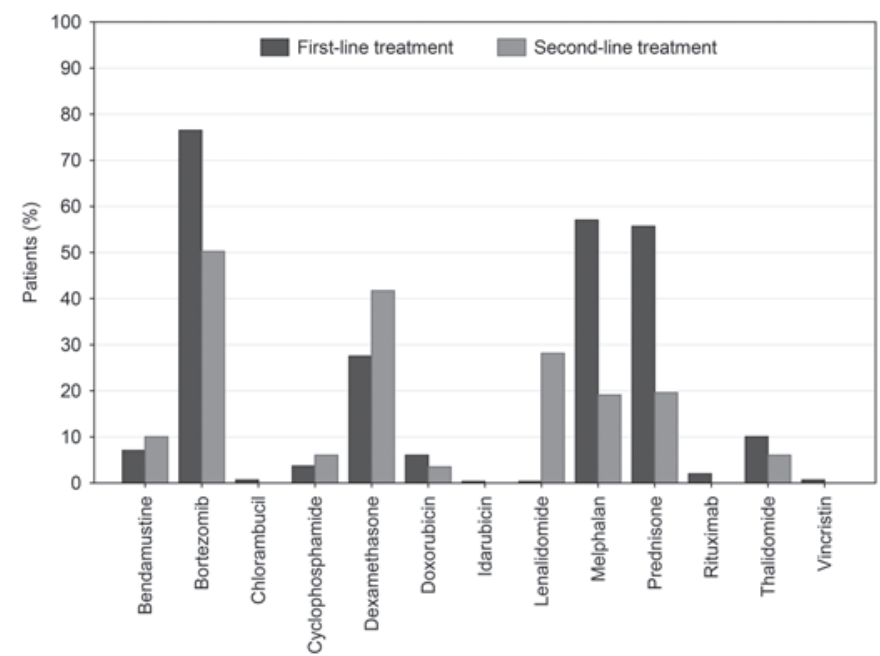

Fig. 3. Frequency of active substances in both treatment lines (first-line treatment: $\mathrm{n}=298$, second-line treatment: $\mathrm{n}=199$ ).

\section{Results}

Sociodemographic and Clinical Characteristics at the Start of First-Line Treatment

Table 1 shows the sociodemographic and clinical characteristics of the patients with MM at the start of their first-line treatment (in total and for the 3 most frequent therapeutic regimens) in detail. Of the 298 patients who were enrolled at the beginning of their first-line systemic treatment, $49 \%$ are male. The median age of the patients is 72 years at the time of primary diagnosis and 73 years (range 41.6-92.0 years) at the onset of first-line systemic treatment. The average body mass index (BMI) of the patients (male and female) is $26 ; 18 \%$ are obese (BMI $>30$, according to the World Health Organisation (WHO) definition). $76 \%$ of the patients have at least 1 comorbidity. The most frequent comorbidities are cardiovascular disorders ( $49 \%$ of the patients) and diabetes $(17 \%) .8 \%$ of the diabetic patients have signs of polyneuropathy, corresponding to $3 \%$ of the whole study population. The average CCI of 0.9 indicates that most patients are in good general condition. The majority of the patients are in stage III (67\%) according to the classification of Durie and Salmon [13], which is identified by clinical features and correlates with shortened OS [13, 14]. In contrast, approximately $8 \%$ of the patients are enrolled in myeloma stage I. B symptoms are present in $13 \%$ of the patients. The predominant systemic symptom is weight loss (in 7\% of all and in $57 \%$ of the affected patients). $19 \%$ of the patients suffer from impaired renal function measured by creatinine levels above the age-adjusted upper limit, and $11 \%$ of all affected patients are diagnosed with terminal renal insufficiency. The reason for the impairment is mostly reported to be tumour related ( $71 \%$ of all affected patients). $31 \%$ of the patients are diagnosed with Bence-Jones proteinuria. $45 \%$ of the patients show normal bone structure or only a solitary osteolysis, while $55 \%$ suffer from advanced bone disease. In $65 \%$ of the patients, the rate of plasma cells in the bone marrow exceeds $30 \%$ (table 1 ).

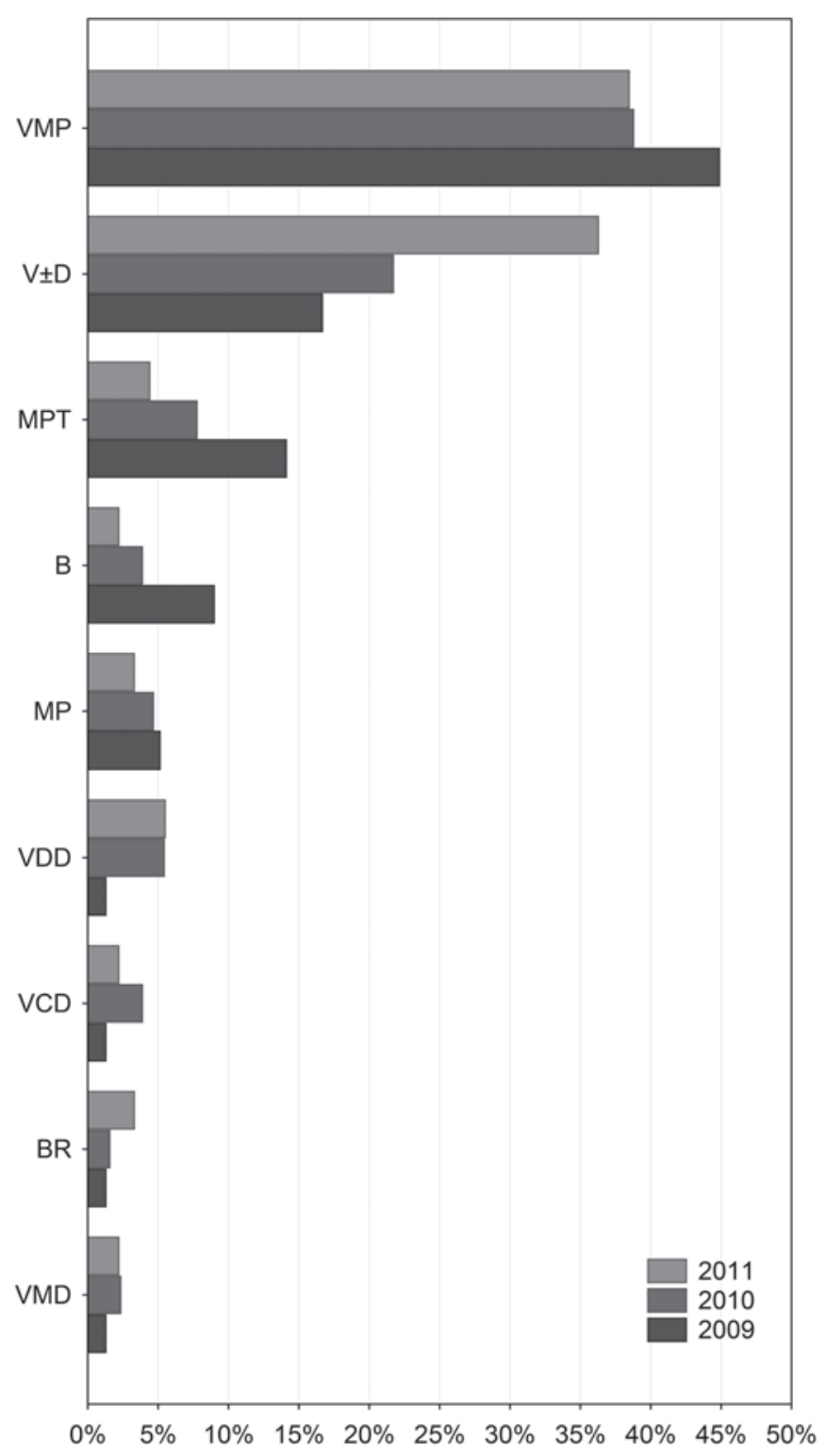

Fig. 4. Frequency of first-line treatment over time $(n=298)$. $B=$ Bendamustine \pm prednisone, $\mathrm{BR}=$ bendamustine + rituximab \pm prednisone, $\mathrm{MP}=$ melphalan + prednisone, $\mathrm{MPT}=$ melphalan + prednisone + thalidomide, $\mathrm{VCD}=$ bortezomib + cyclophosphamide + dexamethasone, $\mathrm{V} \pm \mathrm{D}=$ bortezomib \pm dexamethasone, $\mathrm{VDD}=$ bortezomib + doxorubicine + dexamethasone, $\mathrm{VMD}=$ bortezomib + melphalan + dexamethasone, $\mathrm{VMP}=$ bortezomib + melphalan + prednisone. $\mathrm{n}(8$ months in 2009) $=78$, $\mathrm{n}(12$ months in 2010) $=129, \mathrm{n}(11$ months in 2011$)=91$.

Sociodemographic and Clinical Characteristics at the Start of Second-Line Treatment

In the TLN, the majority of the patients (80\%) were enrolled for first-line treatment whereas $20 \%$ were included for second-line treatment. Table 1 shows in detail the sociodemographic and clinical characteristics of the patients at the start of their second-line treatment (in total and for the 3 most frequent therapeutic regimens). Of the 73 patients who were enrolled in the second line, $48 \%$ are male. The median age of the patients is 73 years at the time of primary diagnosis and 75 


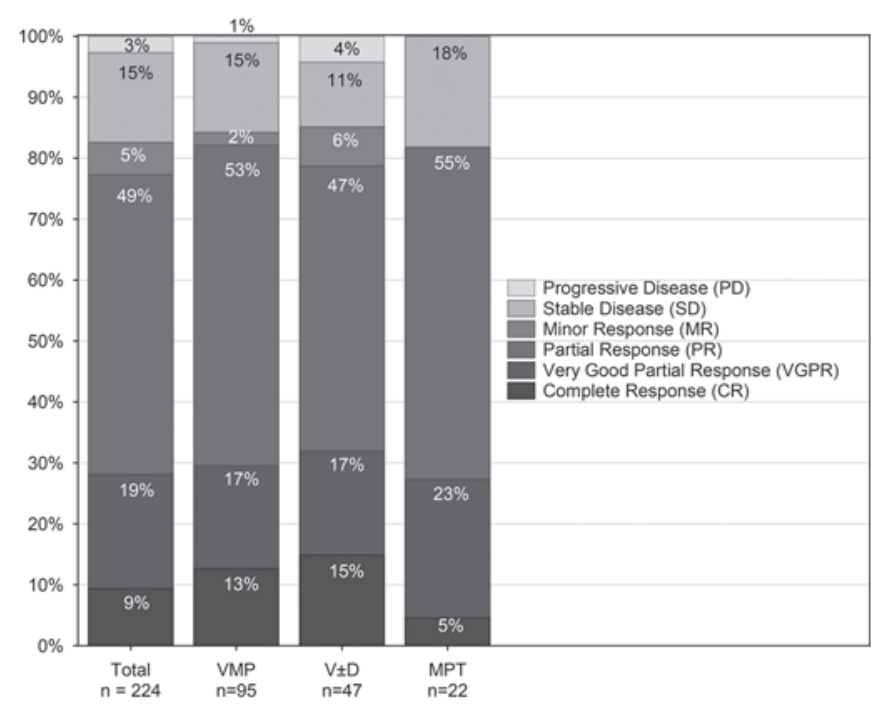

Fig. 5. Best clinical response of first-line treatment (non-transplant patients). Patients with completed first-line treatment and available parameter on best clinical response. MPT $=$ Melphalan + prednisone + thalidomide, $\mathrm{V} \pm \mathrm{D}=$ bortezomib \pm dexamethasone, $\mathrm{VMP}=$ bortezomib + melphalan + prednisone.

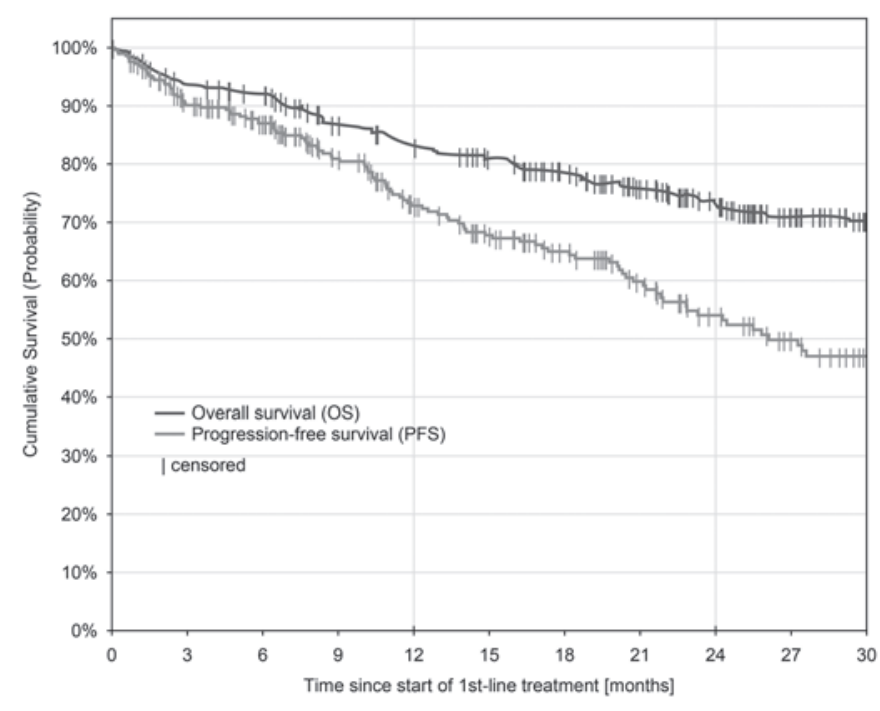

\begin{tabular}{lrllllllllll}
\multicolumn{2}{l}{$\begin{array}{l}\text { Patients at risk } \\
\text { PFS }\end{array} \quad 298$} & 246 & 216 & 177 & 148 & 127 & 109 & 88 & 67 & 54 & 43 \\
OS & 298 & 267 & 256 & 229 & 215 & 201 & 185 & 161 & 135 & 109 & 91
\end{tabular}

Fig. 6. PFS and OS of first-line treatment $(\mathrm{n}=298)$.

years (range 47.3-90.4 years) at the onset of their second-line systemic treatment. The patients' clinical and laboratory parameters are quite comparable to those of the patients enrolled for first-line treatment. Comorbidities are present slightly more often within this collective: $80 \%$ of all patients have at least 1 comorbidity. The rate of cardiovascular disorders among this group is $60 \%$, and $21 \%$ of the patients suffer from diabetes. Altogether, the average CCI of 1.1 indicates that pre-existing medical conditions are rather mild in this cohort, but higher than in patients enrolled for first-line treatment. For more details, see table 1.

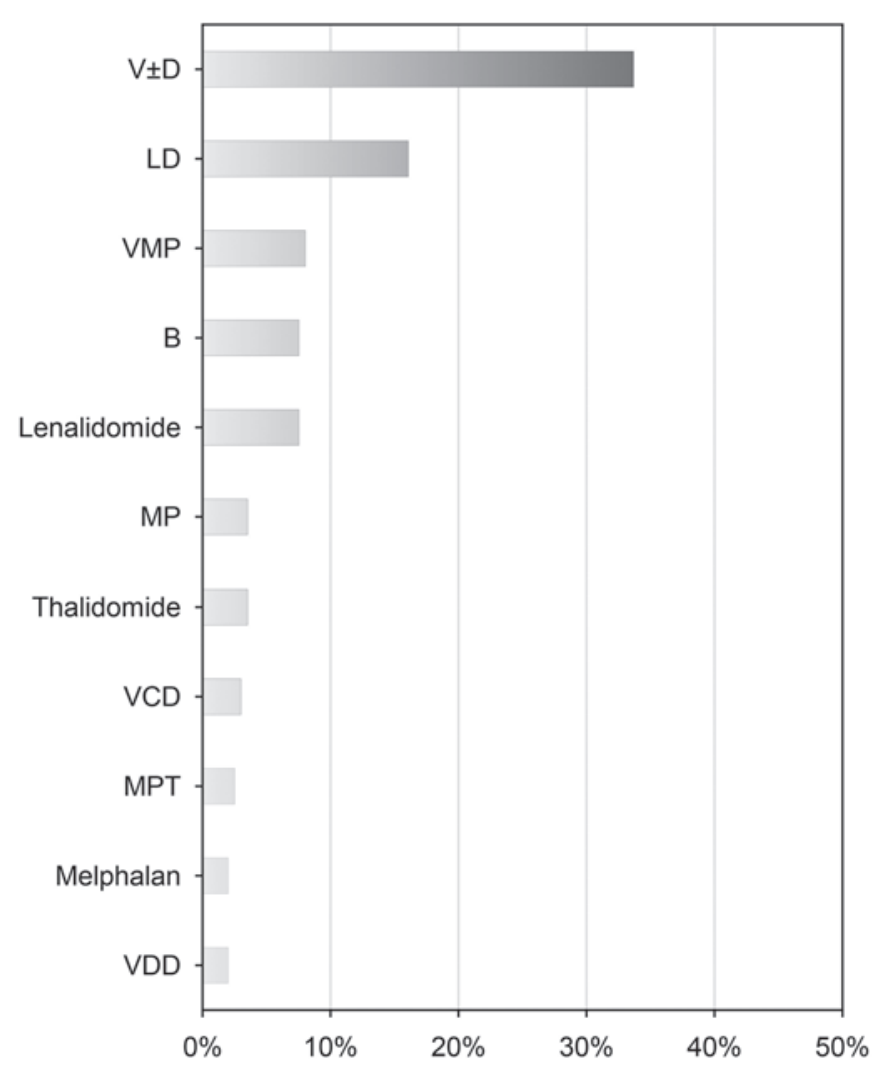

Fig. 7. Frequency of second-line treatment $(\mathrm{n}=199)$. $\mathrm{B}=$ Bendamustine \pm prednisone, $\mathrm{LD}=$ lenalidomide + dexamethasone, $\mathrm{MP}=$ melphalan + prednisone, $\mathrm{MPT}=$ melphalan + prednisone + thalidomide, $\mathrm{VCD}=$ bortezomib + cyclophosphamide + dexamethasone, $\mathrm{V} \pm \mathrm{D}=$ bortezomib \pm dexamethasone, VDD = bortezomib + doxorubicine + dexamethasone, $\mathrm{VMP}=$ bortezomib + melphalan + prednisone.

\section{First-Line Treatment}

Data of 298 first-line treatments are available. With regard to the substances applied in the first line, bortezomib $(\mathrm{V})$ is used in $77 \%(\mathrm{n}=228)$, melphalan $(\mathrm{M})$ in $57 \%(\mathrm{n}=170)$, prednisone $(\mathrm{P})$ in $56 \%(\mathrm{n}=166)$, dexamethasone $(\mathrm{D})$ in $28 \%(\mathrm{n}=$ $82)$ and thalidomide $(\mathrm{T})$ in $10 \%(\mathrm{n}=30)$ of all patients (fig. 3$)$.

The most common regimen is VMP (bortezomib + melphalan + prednisone) administered in overall $40 \%(n=120)$ of the patients. $\mathrm{V} \pm \mathrm{D}$ (bortezomib + dexamethasone) is applied in $25 \%(n=74)$ of the patients while $8 \%(n=25)$ of the patients are treated with MPT (melphalan + prednisone + thalidomide). First-line treatment has changed considerably over time since the start of the registry (fig. 4). While VMP was used in the treatment of $45 \%$ of all patients in 2009 , the rate declined to $38 \%$ in 2011 . In all other combinations, the use of bortezomib increased: $\mathrm{V} \pm \mathrm{D}$ from $17 \%$ to $36 \%$, VDD (bortezomid + doxorubicine + dexamethasone) from $1 \%$ to $5 \%$, and VCD (bortezomib + cyclophosphamide + dexamethasone) and VMD (bortezomib + melphalan + dexamethasone) from $1 \%$ to $2 \%$.

In contrast, the use of melphalan as initial treatment decreased in nearly all combinations: VMP from $45 \%$ to $38 \%$, MPT from $14 \%$ to $4 \%$, and MP (melphalan + prednisone) from $5 \%$ to $3 \%$. 
Table 2. Treatment sequences proceeding from first-line to second-line treatment

\begin{tabular}{|c|c|c|c|c|}
\hline & & & $\mathrm{n}$ & $\%$ \\
\hline Patients & & & 298 & \\
\hline Treatment strategy & & & 126 & 100.0 \\
\hline Bortezomib based & $\rightarrow$ & lenalidomide based & 44 & 34.9 \\
\hline Bortezomib based & $\rightarrow$ & bortezomib based & 33 & 26.2 \\
\hline Bendamustine based & $\rightarrow$ & bortezomib based & 8 & 6.3 \\
\hline Treatment regimen & & & 126 & 100.0 \\
\hline VMP & $\rightarrow$ & LD & 17 & 13.5 \\
\hline VMP & $\rightarrow$ & $\mathrm{V} \pm \mathrm{D}$ & 11 & 8.7 \\
\hline $\mathrm{V} \pm \mathrm{D}$ & $\rightarrow$ & LD & 8 & 6.3 \\
\hline VMP & $\rightarrow$ & lenalidomide & 8 & 6.3 \\
\hline
\end{tabular}

$\mathrm{LD}=$ Lenalidomide + dexamethasone, $\mathrm{V} \pm \mathrm{D}=$ bortezomib \pm dexamethasone, $\mathrm{VMP}=$ bortezomib + melphalan + prednisone.
Altogether, the use of all bortezomib-based regimens increased from $67 \%$ to $85 \%$, while the use of all melphalanbased regimens decreased from $68 \%$ to $48 \%$.

At the time of this analysis, best clinical response was documented in $75 \%(n=224)$ of the first-line treatments (fig. 5); this represents $79 \%$ of all completed first-line treatments $(\mathrm{n}=$ 284). The objective response rate (ORR; encompassing any positive response) was $82 \%$ (fig. 5), with a complete response rate $(\mathrm{CR})$ of $9 \%$ and a (very good) partial or minor response rate $(\mathrm{PR} / \mathrm{MR})$ of $73 \%$. Only in $3 \%$ of the patients, progressive disease (PD) was documented (fig. 5). In particular, the ORR was $85 \%$ in the VMP regimen $(n=95 ; 13 \% \mathrm{CR}, 72 \%$ (very good) $\mathrm{PR}$ or $\mathrm{MR}), 85 \%$ in the $\mathrm{V} \pm \mathrm{D}$ regimen $(\mathrm{n}=47 ; 15 \% \mathrm{CR}$, $70 \%$ (very good) PR or MR) and $82 \%$ in the MPT regimen ( $\mathrm{n}=22 ; 5 \% \mathrm{CR}, 77 \%$ (very good) PR or MR), as documented at one time point during the treatment (fig. 5).

With a median follow-up time of 30 months (maximum 51 months), $38 \%$ of the patients had progressed or died after first-line therapy; $30 \%$ of the patients have died overall. Due to the resulting high number of censored cases, the estimated PFS and OS values still have to be interpreted with caution. Overall, the 2-year PFS was 54\% (1-year PFS: 73\%) and the 2-year OS was 73\% (1-year OS: 83\%) (fig. 6).

\section{Second-Line Treatment}

Data of 199 second-line treatments are available. The substances applied in the second line are shown in figure 3: bortezomib (V, 50\%, $\mathrm{n}=100)$, dexamethasone ( $\mathrm{D}, 42 \%, \mathrm{n}=83$ ), lenalidomide ( $\mathrm{L}, 28 \%, \mathrm{n}=56$ ), prednisone ( $\mathrm{P}, 20 \%, \mathrm{n}=39$ ) and melphalan $(\mathrm{M}, 19 \%, \mathrm{n}=38)$.

The most common regimen is $\mathrm{V} \pm \mathrm{D}$ (fig. 7): Overall, $34 \%$ $(n=67)$ of the patients receive this type of treatment. LD (lenalidomide + dexamethasone) is applied in $16 \%(\mathrm{n}=32)$ of the patients, and $8 \%$ are treated with VMP $(\mathrm{n}=16)$, B (bendamustine \pm prednisone; $\mathrm{n}=15$ ) or lenalidomide monotherapy $(\mathrm{n}=15)$, each.

Table 2 shows the 3 most frequently used sequences proceeding from first-line to second-line treatment. In $35 \%$ of the patients, a bortezomib-based treatment is followed by a lenalidomide-based treatment. Further, $26 \%$ of the patients are treated with a regimen containing bortezomib as retherapy.

The number of second-line treatments is too small to analyse changes in treatment over time. Data on remission rates will be analysed once more patients have received second-line treatment.

\section{Discussion}

Clinical registries provide insight into real-life treatment of real-life patients. They reveal which treatments are used in everyday routine practice and how the choice of treatment changes over time. While strict inclusion and exclusion criteria applied in randomized controlled studies (RCTs) are not applied in registries, any therapy used is upon the discretion of the treating physician and strongly depends on the patients' individual characteristics. This becomes evident here.

For the development of an international staging system (ISS) for MM, the International Myeloma Working Group (IMWG) analysed data of 10,750 previously untreated symptomatic myeloma patients [14] and documented a distribution of patients according to Durie and Salmon of $8 \%$ in stage I, $26 \%$ in stage II and $66 \%$ in stage III. These data are comparable with the rates as documented for the patients with MM in the TLN study. So, the TLN sample reflects typical patients with MM, which is further evident when taking into account the distribution according to ISS [14]. Therefore, the patient sample described here can be regarded as representative.

With the European Medicines Agency (EMA) marketing authorisation for the proteasome inhibitor bortezomib (2004 for relapsed, 2008 for untreated patients) and for the immunomodulatory drugs lenalidomide (2007 for relapsed patients) and thalidomide (2008 for untreated patients), the introduction of these new substances led to new treatment choices. Today, melphalan plus prednisone plus either bortezomib or thalidomide are the new standards in Europe for elderly patients and patients ineligible for autoPBSCT [5]. 
Our data show that, today, bortezomib-based therapies are most frequently used in first-line treatment as well as in second-line treatment. Over the course of time, a shift towards the use of bortezomib in first-line treatment of MM is evident: While the use of bortezomib-based regimens increased from $67 \%$ in 2009 to $85 \%$ in 2011 , the use of melphalan-based regimens decreased from $68 \%$ in 2009 to $48 \%$ in 2011 . A comparable analysis considering second-line treatment of $\mathrm{MM}$ will be done later, since the patient number is currently too low.

The 3 most frequently used regimens cover more than $75 \%$ of all patients. In 2009 these patients were treated with VMP, $\mathrm{V} \pm \mathrm{D}$ or MPT whereas in 2011 the most frequently used regimens were $\mathrm{V} \pm \mathrm{D}$, VMP or VDD. However, since some of the first-line treatments are still ongoing, it cannot be excluded that a subgroup may proceed to a subsequent SCT. Especially $\mathrm{V} \pm \mathrm{D}$ is used frequently as induction therapy prior to SCT [5].

Patients who received an SCT were excluded from the present analysis. A preliminary analysis shows that - as expected - these patients are noticeably younger (median age: SCT 60 years vs. non-SCT 72 years) (data on file). Interestingly, the rate of male patients in the registry differs considerably (SCT $66 \%$ vs. non-SCT $49 \%$ ) (data on file).

Approximately one-third of the patients diagnosed with $\mathrm{MM}$ are asymptomatic upon diagnosis, and active surveillance is an internationally accepted option [5, 6]. Nonetheless, MM remains incurable. So, prolonged OS and improvement in quality of life are today's primary goals of treatment.

Since the introduction of the new substances in 2004-2008, the treatment guidelines have been adapted: The previous socalled standard therapy with vincristine + doxorubicine + dexamethasone (VAD) [15] has been withdrawn from the guidelines and is not recommended anymore [6]. So, it is not surprising that VAD is not among the 10 most frequently used regimens today. The registry started after the EMA granted market authorisation for the new substances; thus, a shift towards these substances cannot be mapped. Due to the heterogeneity of the patient cohorts, future analysis may reveal who might profit most from one particular regimen. It is of great interest to see whether these substances improve OS, especially when used in second-line treatment.

This present analysis focuses on the description of routine treatment. These data may help health authorities in assessing the status of care and deciding on the adaption of new scientific evidence for daily routine. Insurance companies may better calculate their budgets. Furthermore, treating physicians may analyse their own treatment behaviour in the light of the registry data. Besides examining the frequency of specific regimens in different therapy lines, parameters describing their effectiveness in 'real life' are of great importance. These analyses need time.

At the time of this analysis, the majority of the patients were alive and without progression, limiting the reliability of the Kaplan-Meier survival estimates. Nevertheless, considering these limitations and the fact that we present non-trans- plant patients with comorbidities, the estimated 1- and 2-year PFS and OS data compare favourably with data published in recent clinical trials $[16,17]$. The high proportion of censored cases precluded comparative analyses of the effectiveness of various first-line regimens by multivariate regression models.

The interpretation of effectiveness in 'real life' is substantially more complex than that of efficacy in RCTs, since the patient characteristics are much more heterogeneous than in controlled trials. These aspects will be of central interest for the TLN in the future.

\section{Acknowledgements}

The authors would like to thank all members of the TLN Study Group and all patients at the study sites for their participation in the study. Special thanks go to Martina Jänicke and Victoria Smith-Machnow for their review and support with the manuscript and to Johanna Harde for updating the data of the original manuscript. The TLN Study Group collaborates with the Arbeitskreis Klinische Studien (AKS) and the Kompetenznetz Maligne Lymphome (KML).

\section{Authorship and Contribution}

N.M. and W.K. conceptualised and designed the registry. R.G. coordinates the registry. W.K., R.G. and H.H. elaborated the concept for this paper, analysed and interpreted the data and wrote the manuscript. M.M. did the statistical analysis and generated the figures. W.K., W.A., A.A., A.N. and N.M. recruited patients and collected and documented data. All authors reviewed and approved the submitted manuscript.

\section{Participating Study Sites (Principal Investigator, Location)}

Abenhardt, München; Aldaoud, Leipzig; Anhuf, Duisburg; Bakhshandeh-Bath, Hamburg; Becker, Porta Westfalica-Barkhausen; Bertram, Hamburg; Bruch, Bonn; Buschmann, Rheinbach; Decker, Ravensburg; Dörfel, Dresden; Elsel, Zwickau; Engel, Hamburg; Eschenburg, Güstrow; Fiechtner, Stuttgart; Fietz, Singen; Fries, Bamberg; Groschek, Würselen; Hahn, Herne; Hansen, Kaiserslautern; Harich, Hof; Hegener-Tschochner, Düsseldorf; Hegewisch-Becker, Hamburg; Höring, Stuttgart; Hurtz, Halle; Hutzschenreuter, Nordhorn; Illmer, Dresden; Janssen, Westerstede; Josten und Klein, Wiesbaden; Kingreen, Berlin; Knauf, Frankfurt; Köchling, Villingen-Schwenningen; Koenigsmann, Hannover; Köhler, Langen; Kremers, Lebach; Kröger, Bremerhaven; Ladda, Neumarkt; Lerchenmüller, Münster; Linck, Euskirchen; Lipke, Dortmund; Losem, Neuss; Marquard, Celle; Meyer, Berlin; Michl, München; Mittermüller, Germering; Müller, Leer; Nawka, Berlin; Neise, Krefeld; Nusch, Velbert; Otremba, Oldenburg; Overkamp, Recklinghausen; Petersen, Heidenheim; Priebe-Richter, Stadthagen; Rauh, Witten; Reiser, Köln; Rendenbach, Trier; Rodemer, Wilhelmshaven; Rubanov, Hameln; Sandner, Passau; Sauer, Potsdam; Schlag, Würzburg; Schmidt, München; Schneider-Kappus, Ulm; Schnell, Frechen; Schröder, Mülheim a. d. Ruhr; Schulz, Bad Kreuznach; Schulze, Zittau; Schwindt, Bonn; Seipelt, Bad Soden; Seraphin, Northeim; Slawik, Augsburg; Söling, Kassel; Stoetzer, München; Tamm, Berlin; Tessen, Goslar; Tschechne, Lehrte; Vehling-Kaiser, Landshut; von Verschuer, Essen; von Wussow, Hannover; Weber, Homberg (Efze); Weide, Koblenz; Welslau, Aschaffenburg; Wierecky, Hamburg; Zaiss, Freiburg. 


\section{Trial Registration}

ClinicalTrial.gov registry NCT00889798.

\section{Funding}

The TLN Registry is funded by iOMEDICO. The analysis was supported by Janssen-Cilag GmbH.

\section{Disclosure Statement}

The authors report no potential conflicts of interest.

Online Supplemental Material

Supplemental Table 1. Patients' characteristics at time of enrolment To access the online supplemental material please refer to $w w w . k a r g e r$. com/?DOI=000368315.

\section{References}

1 Kyle RA, Rajkumar SV: Multiple myeloma. Blood 2008;111:2962-2972.

2 GLOBOCAN 2008, globocan.iarc.fr/ [cited April 27, 2012].

3 Robert Koch-Institut, Gesellschaft der epidemiologischen Krebsregister in Deutschland e.V. (eds): Krebs in Deutschland 2007/2008, ed 8. Berlin, Robert Koch-Institut, 2012

4 Boyle P, Ferlay J: Cancer incidence and mortality in Europe, 2004. Ann Oncol 2005;16:481-488.

$\checkmark 5$ Harousseau J-L, Dreyling M; on behalf of the ESMO Guidelines Working Group: Multiple myeloma: ESMO clinical practice guidelines for diagnosis, treatment and follow-up. Ann Oncol 2010;21:v155-v157.

6 Kortüm M, Einsele H, Naumann R, Peest D, Liebisch P, Goldschmidt H: DGHO-Leitlinie Multiples Myelom 2010. www.dgho-onkopedia.de/de/onkopedia/leitlinien/leitlinien_overview, 2010.

7 National Comprehensive Cancer Network $(\mathrm{NCCN})$ : NCCN guidelines: multiple myeloma. www.nccn.org, 2013

8 Bundesärztekammer:Tätigkeitsbericht 2011 der Bundesärztekammer [Activity report of the German Medical Association 2011]. www.bundesaerztekammer.de downloads/Taetigkeit2011.pdf, Nürnberg, 2012.
9 Berufsverband der Niedergelassenen Hämatologen und Onkologen in Deutschland (BNHO) e.V.: Qualitätsbericht der onkologischen Schwerpunktpraxen 2010 [Quality report of the oncology outpatient-centers 2009]. www.winho.de/fileadmin/2012/ Qualitaetsbericht_2010.pdf, 2011.

10 Charlson ME, Pompei P, Ales KL, MacKenzie CR: A new method of classifying prognostic comorbidity in longitudinal studies: development and validation. J Chronic Dis 1987;40:373-383.

Kaplan EL, Meier P: Nonparametric estimation from incomplete observations. J Am Stat Assoc 1958:53:457-481.

12 Schemper M, Smith TL: A note on quantifying follow-up in studies of failure time. Control Clin Trials 1996;17:343-346.

13 Durie BG, Salmon SE: A clinical staging system for multiple myeloma. Correlation of measured myeloma cell mass with presenting clinical features, response to treatment, and survival. Cancer 1975;36: 842-854.

14 Greipp PR, San Miguel J, Durie BGM, Crowley JJ, Barlogie B, Bladé J, et al.: International staging system for multiple myeloma. J Clin Oncol 2005; 23:3412-3420.
15 Liebisch P, Peest D: DGHO-Leitlinie Multiples Myelom 2007. www.dgho-onkopedia.de/de/onkopedia/ leitlinien/leitlinien overview, 2007.

16 Mateos M-V, Richardson PG, Schlag R, Khuageva NK, Dimopoulos MA, Shpilberg O, et al.: Bortezomib plus melphalan and prednisone compared with melphalan and prednisone in previously untreated multiple myeloma: updated follow-up and impact of subsequent therapy in the phase III VISTA trial. J Clin Oncol 2010;28:2259-2266.

17 Pönisch W, Mitrou PS, Merkle K, Herold M, Assmann M, Wilhelm G, et al.: Treatment of bendamustine and prednisone in patients with newly diagnosed multiple myeloma results in superior complete response rate, prolonged time to treatment failure and improved quality of life compared to treatment with melphalan and prednisone - a randomized phase III study of the East German Study Group of Hematology and Oncology (OSHO). J Cancer Res Clin Oncol 2006;132:205-212. 